\title{
HomeCare, Elder People Monitoring System and TV Communication
}

\author{
Victor Parra ${ }^{1}$, Vivian López ${ }^{1}$, and Mohd Saberi Mohamad ${ }^{2}$ \\ ${ }^{1}$ Department of Computer Science and Automation, University of Salamanca \\ Plaza de la Merced, s/n, 37008, Salamanca, Spain \\ \{parra, vivian\}@usal.es \\ ${ }^{2}$ Center for Information and Communication Technology (CICT), \\ Universiti Teknologi Malaysia, 81310 UTM Skudai, Johor, Malaysia \\ saberi@utm.my
}

\begin{abstract}
For seniors who require continuous care and do not have the resources to have an assistant continuously, have a low cost system that monitors their environment allows them to have independence, while moving in a secure environment. In addition, accessing to basic services through a platform accessible to all people, including TV, facilitates their integration into the online society.
\end{abstract}

Keywords: Context aware system, multi-agent system, Wireless sensor networks.

\section{$1 \quad$ Introduction}

Today, access to information and numerous services is clearly geared to the network. Thanks to cheaper mobile devices, as well as connections to the Internet and social networks we live interconnected almost 24 hours. However, despite the popularity of touchscreens, smartphones, tablets, etc., communication interfaces with electronic devices remain relatively traditional, as they are geared mostly to young or adult audience accustomed to working with them, leaving outside this range a large sector, such as the elderly. In a society that lives permanently connected, older people who cannot fully care for themselves need personal assistance or admission to a residence, situations that cannot always afford. The availability of a system that would allow them to get in touch with the outside world in a simple way from home [4] facilitate the independence of this sector of society, he could feel more useful, while they would be remotely monitored by experts and can be addressed quickly if necessary.

In this paper a system that is able to monitor a house and manage communication through television, as input interface using the four colors that the remote control is presented. By installing non-intrusive sensors and a low cost unit, a control of the areas in which the person is moving is maintained as well as possible changes in their routine. Moreover, through the television, they can offer personal services to facilitate tasks such as booking appointment at the physician or transport to a day center. 
This article is divided into the following sections: Section 2 describes a state of the art; Section 3 presents the model and the proposed architecture; Section 4 presents the results and conclusions, respectively.

\section{Background}

Today's automation systems are geared mostly to home security, so are oriented mostly to access through doors and windows, as well as the use of cameras and microphones, which can sometimes seem intrusive for tenants. However, a proper arrangement of the sensors in the home can provide much more information. With the present sensors including low cost can be extracted activity patterns [1] that allow additional routines in order to increase safety and energy savings. Not only restricted to housing this possibility, but also the information is accessible remotely [2 3, 17] enabling act if abnormalities in routine. It is especially useful for a routine of an elderly person in your home [4] without a 24/7 assistant. Including fall sensors can even detect emergencies immediately $[5,29,30,31,32,33]$ without intrusive sensors such as cameras.

However, these systems either behave reactively or just extract information to define the behavior. The interaction with a human being usually takes place via computer or Smartphone, which usually requires some knowledge of handling such devices. Smart TV systems menus provide access to services that require sailings not always intuitive menus. This makes people not related to new technologies, such as the sector of the elderly, and not able to enjoy access to online services. Projects like [7] propose the use of television as a tool to integrate people with reduced abilities, being the sole mechanism for the provision of services and obtaining information, without access to the monitoring of the person. On the other hand, the European project Monami proposes a comprehensive framework to provide services to elderly and disabled people, focusing on providing services to foster a more independent life style. However, it provides no information to Physicians or alarm systems.

\section{Proposed Reasoning System}

The system proposed in this paper combines the collection of data from distributed sensors in a home for a routine of a person and to detect any abnormalities caused by a problem. The system would permanently be connected to a medical center, which would receive an alert with the conditions given. Thus, a person would not require admission to a residence or the need for a $24 / 7$ wizard. The doctor may communicate directly with the person to carry a medical control of it in real time. Furthermore, we propose the use of television as a method of two-way interaction, so that the person could receive information through it, as well as reminders on taking pills, and also may request certain services, such as an appointment with Physician, transport to the reserve day or a food menu. Communication with television posed by the remote control of television, taking advantage that it is a physical interface friendly and known for virtually all of the current population to full. 
Agents and multi-agent systems have been successfully implemented in areas such as e-commerce, medicine, oceanography, robotics, etc. $[15.16,18,19,20,21,22,23$, $24,25,26,27,28]$. To perform the operation of the entire system, use of multi-agent architecture is PANGEA [10, 34], on which different organizations have been included in order to delegate responsibilities and facilitate the distribution and decentralization. This allows us to divide the system into a smaller, easier to maintain and to update sections. Fig. 1 shows the organization created, only the roles associated to the system not including PANGEA own account. As one can see it is the control that handles sensors monitor the sensor value changes and react to these organization. The agent communication is responsible for managing notifications, communication and user interaction with the system.

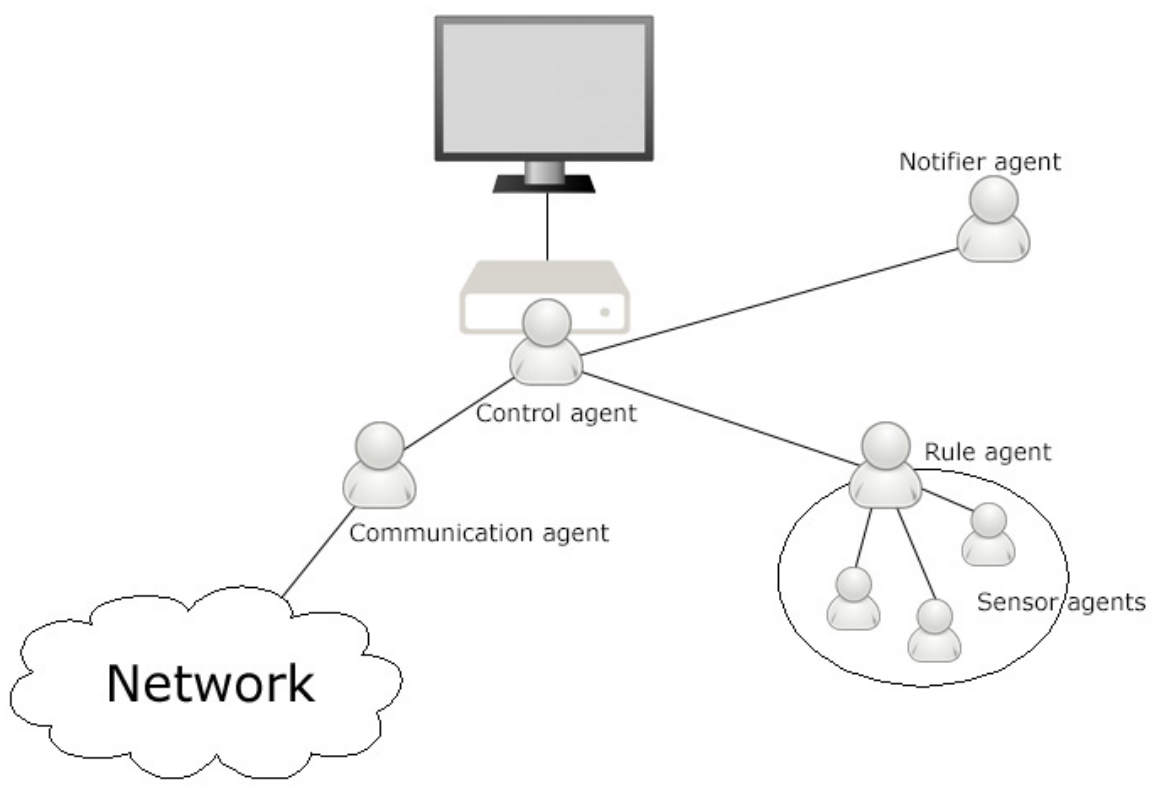

Fig. 1. Agent diagram

\subsection{Sensor Control}

On the one hand, the sensors are read by agents dedicated to them, making the readings periodically and storing the information. Each of these agents would monitor a sensor such that it can detect a failure in the reading or a change in value in the same. If this anomaly or change detected, you will notify the agent that plays the role of management rules, which apply an order based on the rules. These rules are grouped into a "library" that defines a typical daily activity plan. An example would be "If the bed sensor is active for more than eight hours, an alert is generated." To configure the Drools rules engine is used [8], which is a system for managing business rules (known in English as BRMS Business Rule Management System) with a rules engine based on forward chaining inference, using an object-oriented 
implementation of the Rete algorithm [9]. Thus, the semantics is exposed using the domain specific language (DSL). The re-icing are expressed by the Drools rule language, called DRL that allows you to embed Java code or MVEL for the implementation of the rules. It also supports the ability to write in EXCEL files. An example of a simple rule, with integrated Java code could be next:

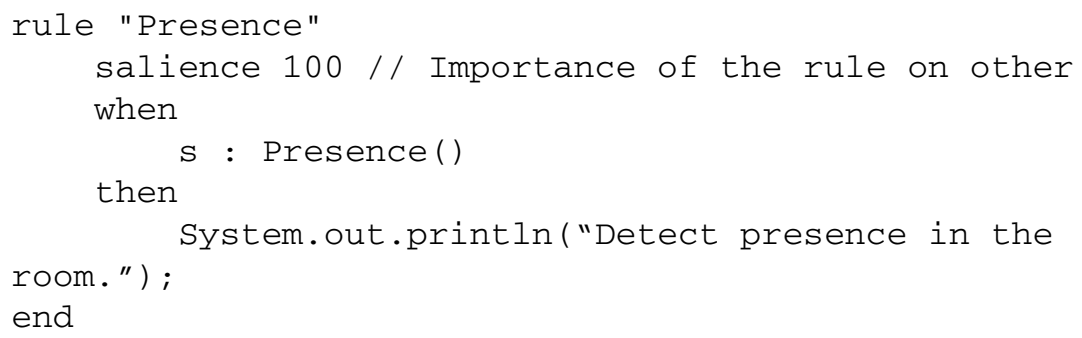

Having a number of sensors in the various rooms of the house allows to control the routine of the older person and their usual tasks in a non-intrusive way. The data obtained by the system and can be processed to extract a set routine that allows key points on which a problem has more or less weight of importance if it occurs. However, the communication application can operate even without any sensor, thus providing a low-cost accessible to any person wishing to use.

The sensor array is not restricted to a particular type, they may be present, pressure, temperature, humidity, etc... The main advantages of these sensors allow obtaining reliable data with minimum power consumption and low cost. This allows the installation of sensors in wireless modules, avoiding the need for a cable installation throughout the home. To this end, are connected with the ZigBee radio modules [11], whose technology allows high battery life without recharging it in months.

PIR sensors (Fig. 2) allow greater battery saving since only consumed when detected emitted radiation by the human body, which activates the sensor circuit, consuming power only in that moment.

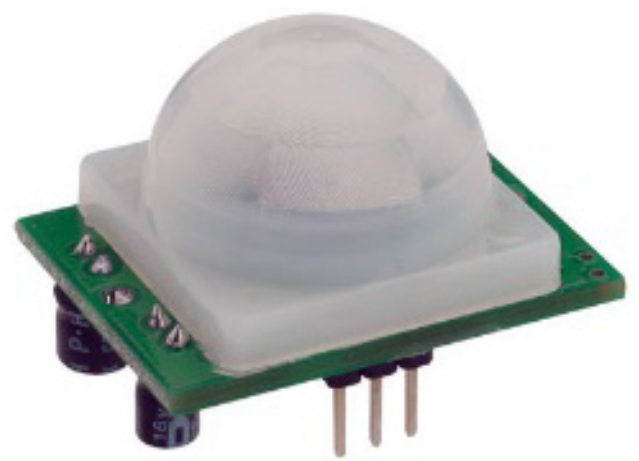

Fig. 2. Presence sensor PIR 
Moreover, pressure sensors allow to obtain a reading of places where the person usually is, as for example in the bed, so that an activation of the sensor for more than $\mathrm{X}$ times could be a problem. A more detailed description about the sensor features can be seen in this previous work 12 .

\subsection{User Control Events}

Moreover, the control agent role is in the computer connected to the TV and is in charge of collecting the throwing events the user through the remote control, in addition to displaying menus and alerts issued by the agents and notification rules (which manages periodic alerts or reminders). The Notification Agent is responsible for managing the user-programmed or assistant alerts, such as taking pills, when the doctor's appointment, etc.

The local exchange is a low cost computer and very low power consumption, so it just means a significant investment and saving the high costs of ongoing care of an elderly person. This unit only requires a power internet access and an HDMI connection to the TV (Fig. 3).

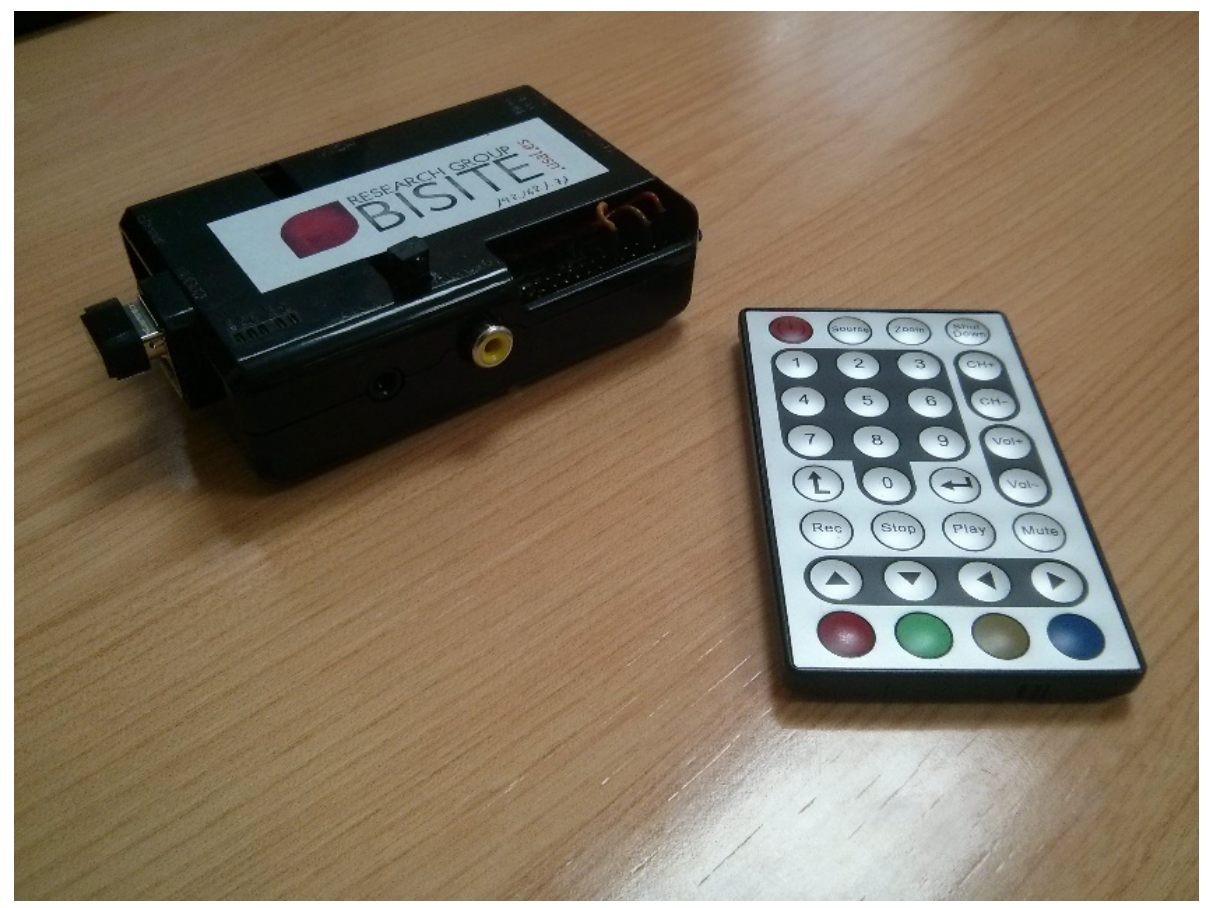

Fig. 3. Raspberry PI model B

The HDMI connection allows two-way communication device to the TV, making it unnecessary to use an additional command to access the available services. Also, the system is able to control the channel tuning, allowing the display off warnings even when the person is watching TV. These notifications would be monitor and reminded, 
making the automatic agenda. If you do not have a TV with HDMI connection, the system carries an infrared receiver that allows the mapping of the TV's remote, which recognizes recorded codes and would be associated with key management options. This protocol uses CEC (Consumer Electronics Control) which is supported by a variety of manufacturers. It allows the user to control and monitor up to ten CEC devices connected via HDMI using a single remote. The CEC protocol uses a single bidirectional wire serial bus for communication. As an example of important commands we have: to activate a device which is in standby status, playback control, tuning menus and other devices. Since the target audience to whom the purpose of this article is geared is mainly old people, it is intended the control system to be as simple and intuitive as possible. Thus, it is proposed to use only the colored buttons of the remotes, which allow the creation of graphical interfaces that can be associated immediately with the option to select. The entire system is designed with the aim that no more buttons are needed and they should be as simple as possible and also they should get rid off of unnecessary navigation between levels in menus.

If the TV does not have HDMI connection, the system carries an infrared receiver that will map the remote control codes, so it can also be used without the need to incorporate additional control.

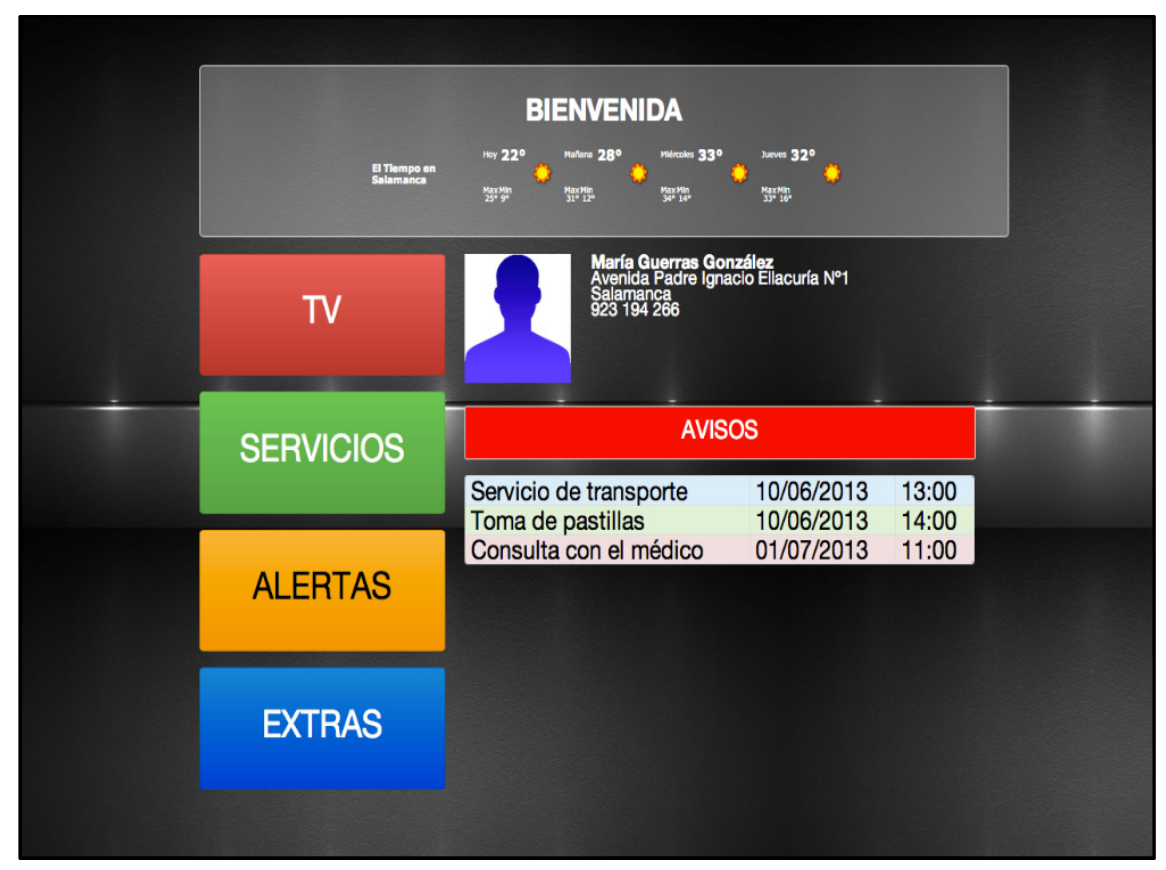

Fig. 4. Main screen

The communication interface consists only of the four colored buttons (red, green, yellow and blue) that provide specific functionality for each screen. The red button is associated in all cases with the "back" so that it becomes associated immediately. On the home screen, as it can be seen in Fig. 4, the main menu provides access to the offered services as well as viewing alerts or add-ons, which are rated with lower priority. Among 
the available services, one of the most important can automatically make an appointment with the doctor. The user simply selects the option, the system takes care of the hospital contact and booking appointment, getting the user both a visual confirmation by the monitor and a call phone. Similarly, according to the appointment date approaches, the user receives reminder alerts on your television character. This feature is especially geared to seniors who are in early stages of Alzheimer's disease.

Regardless of reminder alerts, you can schedule automatic alerts or even perform them manually, with the goal of achieving an active monitoring. Thus, you can control whether you have taken medication rightful, and get the answer that the user transmits through the remote control. This system is also used for the detection of alarms in the house itself, if the person has a sensor on the door to notify you when you leave it open, or fall sensor, which would first alert asking the user if you are alright. All management of alarms based on the value of the sensors is performed as indicated in Section 3.2. Failure to receive a response within a specified time, the system considers that the person is not able to reach the control, so it would launch a medical alert service.

\section{$4 \quad$ Results and Conclusions}

The obtained results with the system have shown that integration with older people has been completely successful. The use of colors when associating with the actions of the menus has allowed a very steep learning curve, and the combination of red button with the option to back the more easily accepted. On the other hand, access of older people to the possibility of requesting services instantly without the need of relying on a phone has enabled them to increase their sense of individualism and independence. The system of reminders for taking medication or transportation schedules doctor's appointment and avoid the need to constantly check the schedules, managing largely dispel the feeling of anxiety.

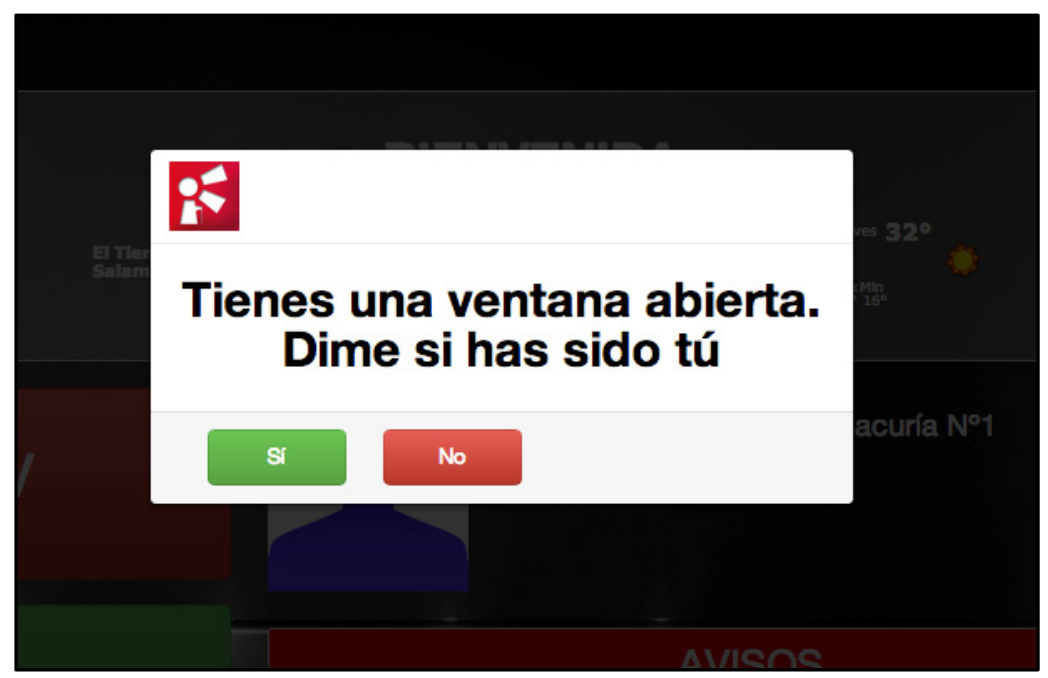

Fig. 5. Alert 
Moreover, the use of low-cost devices allows installation in homes at an affordable price, being more relevant the cost of internet connection as the system itself. This allows the installation of a monitoring system for the elderly without the high cost of entry into a residence or a $24 / 7$ assistant.

The rules engine allows to modify the behavior of agents at runtime without requiring the platform stopping for behavior modification. In future work, agents for the automatic learning will be added, allowing the detection of anomalous situations by implementing the function regarding the sensor values. Formerly already been done some research in this area [13].

Acknowledgments. Work partially supported by the Spanish Government through the project iHAS (grant TIN2012-36586-C03).

\section{References}

1. Tapia, E.M., Intille, S.S., Larson, K.: Activity recognition in the home using simple and ubiquitous sensors. In: Ferscha, A., Mattern, F. (eds.) PERVASIVE 2004. LNCS, vol. 3001, pp. 158-175. Springer, Heidelberg (2004)

2. Noury, N., Hervé, T., Rialle, V., Virone, G., Mercier, E., Morey, G., Porcheron, T.: Monitoring behavior in home using a smart fall sensor and position sensors. In: 1st Annual International Conference on Microtechnologies in Medicine and Biology, pp. 607-610. IEEE (2000)

3. Lymberopoulos, D., Bamis, A., Savvides, A.: Extracting spatiotemporal human activity patterns in assisted living using a home sensor network. Universal Access in the Information Society 10(2), 125-138 (2011)

4. Noury, N.: A smart sensor for the remote follow up of activity and fall detection of the elderly. In: 2nd Annual International IEEE-EMB Special Topic Conference on Microtechnologies in Medicine \& Biology, pp. 314-317. IEEE (2002)

5. Noury, N., Fleury, A., Rumeau, P., Bourke, A.K., Laighin, G.O., Rialle, V., Lundy, J.E.: Fall detection-principles and methods. In: 29th Annual International Conference of the IEEE Engineering in Medicine and Biology Society, EMBS 2007, pp. 1663-1666. IEEE (August 2007)

6. Rao, B.P., Saluia, P., Sharma, N., Mittal, A., Sharma, S.V.: Cloud computing for Internet of Things \& sensing based applications. In: 2012 Sixth International Conference on Sensing Technology (ICST), pp. 374-380. IEEE (December 2012)

7. Alonso, R.S., Tapia, D.I., Villarrubia, G., De Paz, J.F.: Agent technology and wireless sensor networks for monitoring patients in residences and their homes. In: Corchado, J.M., et al. (eds.) PAAMS 2013. CCIS, vol. 365, pp. 417-428. Springer, Heidelberg (2013)

8. Drools. The Business Logic Integration Platform, http: / / www . jboss .org/drools /

9. Berstel, B.: Extending the RETE algorithm for event management. In: On Proceedings of Ninth International Symposium on Temporal Representation and Reasoning, TIME 2002 (2002)

10. Sánchez, A., Villarrubia, G., Zato, C., Rodríguez, S., Chamoso, P.: A Gateway Protocol Based on FIPA-ACL for the New Agent Platform PANGEA. In: Pérez, J.B., et al. (eds.) Trends in Pract. Appl. of Agents \& Multiagent Syst. AISC, vol. 221, pp. 41-51. Springer, Heidelberg (2013) 
11. ZigBee Standards Organization: ZigBee Specification Document 053474r13. ZigBee Alliance (2006)

12. Alonso, R.S., Tapia, D.I., Bajo, J., García, Ó., de Paz, J.F., Corchado, J.M.: Implementing a hardware-embedded reactive agents platform based on a service-oriented architecture over heterogeneous wireless sensor networks 11(1), 151-166 (January 2013)

13. de Paz, J.F., Alonso, R.S., Tapia, D.I.: A Case-Based Planning Mechanism for a Hardware-Embedded Reactive Agents Platform. In: Casillas, J., Martínez-López, F.J., Corchado, J.M. (eds.) Management of Intelligent Systems. AISC, vol. 171, pp. 121-130. Springer, Heidelberg (2012)

14. Bajo, J., Corchado, J.M.: Evaluation and monitoring of the air-sea interaction using a CBR-agents approach. In: Muñoz-Ávila, H., Ricci, F. (eds.) ICCBR 2005. LNCS (LNAI), vol. 3620, pp. 50-62. Springer, Heidelberg (2005)

15. Bajo, J., De Paz, J.F., Rodríguez, S., González, A.: Multi-agent system to monitor oceanic environments. Integrated Computer-Aided Engineering 17(2), 131-144 (2010)

16. Borrajo, M.L., Baruque, B., Corchado, E., Bajo, J., Corchado, J.M.: Hybrid neural intelligent system to predict business failure in small-to-medium-size enterprises. International Journal of Neural Systems 21(04), 277-296 (2011)

17. Carneiro, D., Castillo, J., Novais, P., Fernández-Caballero, A., Neves, J.: Multimodal Behavioural Analysis for Non-invasive Stress Detection. Expert Systems With Applications 39(18), 13376-13389 (2012) ISSN: 0957-4174

18. Corchado, J.M., Aiken, J.: Hybrid artificial intelligence methods in oceanographic forecast models. IEEE Transactions on Systems, Man, and Cybernetics, Part C: Applications and Reviews 32(4), 307-313 (2002)

19. Corchado, J.M., De Paz, J.F., Rodríguez, S., Bajo, J.: Model of experts for decision support in the diagnosis of leukemia patients. Artificial Intelligence in Medicine 46(3), 179-200 (2009)

20. Corchado, J.M., Bajo, J., De Paz, J.F., Rodríguez, S.: An execution time neural-CBR guidance assistant. Neurocomputing 72(13), 2743-2753 (2009); De Paz, J.F., Rodríguez, S., Bajo, J., Corchado, J.: Case-based reasoning as a decision support system for cancer diagnosis: A case study. International Journal of Hybrid Intelligent Systems 6(2), 97-110 (2009)

21. Fdez-Riverola, F., Corchado, J.M.: CBR based system for forecasting red tides. Knowledge-Based Systems 16(5), 321-328 (2003)

22. Fraile, J.A., Bajo, J., Corchado, J.M., Abraham, A.: Applying wearable solutions in dependent environments. IEEE Transactions on Information Technology in Biomedicine 14(6), 1459-1467 (2011)

23. Garijo, F., Gómez-Sanz, J.J., Pavón, J., Massonet, P.: Multi-agent system organization: An engineering perspective. Pre-Proceeding of the 10th European Workshop on Modeling Autonomous Agents in a Multi-Agent World (MAAMAW 2001) (2001)

24. Griol, D., García-Herrero, J., Molina, J.M.: Combining heterogeneous inputs for the development of adaptive and multimodal interaction systems. Advances in Distributed Computing and Artificial Intelligence Journal

25. Pavon, J., Sansores, C., Gomez-Sanz, J.J.: Modelling and simulation of social systems with INGENIAS. International Journal of Agent-Oriented Software Engineering 2(2), 196-221 (2008)

26. Pinzón, C.I., Bajo, J., De Paz, J.F., Corchado, J.M.: S-MAS: An adaptive hierarchical distributed multi-agent architecture for blocking malicious SOAP messages within Web Services environments. Expert Systems with Applications 38(5), 5486-5499 
27. Rodríguez, S., Pérez-Lancho, B., De Paz, J.F., Bajo, J., Corchado, J.M.: Ovamah: Multiagent-based adaptive virtual organizations. In: 12th International Conference on Information Fusion, FUSION 2009, pp. 990-997 (2009)

28. Rodríguez, S., de Paz, Y., Bajo, J., Corchado, J.M.: Social-based planning model for multiagent systems. Expert Systems with Applications 38(10), 13005-13023 (2011)

29. Sánchez-Pi, N., Carbó, J., Molina, J.M.: A Knowledge-Based System Approach for a Context-Aware System. Knowledge-Based Systems 27, 1-17 (2012)

30. Tapia, D.I., Alonso, R.S., De Paz, J.F., Corchado, J.M.: Introducing a distributed architecture for heterogeneous wireless sensor networks. In: Omatu, S., Rocha, M.P., Bravo, J., Fernández, F., Corchado, E., Bustillo, A., Corchado, J.M. (eds.) IWANN 2009, Part II. LNCS, vol. 5518, pp. 116-123. Springer, Heidelberg (2009)

31. Tapia, D.I., Abraham, A., Corchado, J.M., Alonso, R.S.: Agents and ambient intelligence: case studies. Journal of Ambient Intelligence and Humanized Computing 1(2), 85-93 (2010)

32. Tapia, D.I., Rodríguez, S., Bajo, J., Corchado, J.M.: FUSION@, a SOA-based multi-agent architecture. In: International Symposium on Distributed Computing and Artificial Intelligence 2008 (DCAI 2008), pp. 99-107 (2008)

33. Tapia, D.I., Alonso, R.S., García, Ó., de la Prieta, F., Pérez-Lancho, B.: Cloud-IO: Cloud Computing Platform for the Fast Deployment of Services over Wireless Sensor Networks. In: Uden, L., Herrera, F., Bajo, J., Corchado, J.M. (eds.) 7th International Conference on KMO. AISC, vol. 172, pp. 493-504. Springer, Heidelberg (2013)

34. Zato, C., et al.: PANGEA - platform for automatic coNstruction of orGanizations of intElligent agents. In: Omatu, S., Paz Santana, J.F., González, S.R., Molina, J.M., Bernardos, A.M., Rodríguez, J.M.C. (eds.) Distributed Computing and Artificial Intelligence. AISC, vol. 151, pp. 229-240. Springer, Heidelberg (2012) 